The Journal of

Mathematics and Computer Science

Available online at

\title{
http://www.TIMCS.com
}

The Journal of Mathematics and Computer Science Vol .3 No.1 (2011) 71-79

\section{Linear System of Equations with Trapezoidal Fuzzy Numbers}

\author{
S.H. Nasseri ${ }^{1, *}$ and M. Gholami ${ }^{2}$ \\ ${ }^{1}$ Department of Mathematics, Mazandaran University, \\ Babolsar, Iran. \\ 2 Department of Mathematics, University of Tarbiat Moallem, \\ Sabzevar, Iran.
}

Received: March 2011, Revised: May 2011

Online Publication: July 2011

\begin{abstract}
A general fuzzy linear system of equations is investigated using embedding approach. In the literature of fuzzy systems two types of linear systems are more important: 1) Fuzzy Linear Systems, 2) Fully Fuzzy Linear Systems. In both class of these systems usually the authors considered triangular type of fuzzy numbers. In this paper, we introduce a linear system of equations with trapezoidal fuzzy numbers as an extension of the fuzzy linear systems which was first introduced by Friedman et al. [6]. Conditions for the existence of a trapezoidal fuzzy solution to $n \times n$ linear system are derived and a numerical procedure for calculating the solution is illustrated with some examples.
\end{abstract}

Keywords: Fuzzy linear system, Embedding approach, Triangular and trapezoidal fuzzy numbers, Trapezoidal fuzzy linear system.

\section{Introduction}

A general model for solving a fuzzy linear system whose coefficients matrix is crisp and the right-hand side column is an arbitrary fuzzy vector, was first proposed by Friedman et al. [6]. Several algorithms for solving fuzzy linear systems have been introduced by many authors $[1,2,3,4,5,7]$. Allahviranloo $[2,3]$ considered the iterative Jacobi, Gauss Siedel method, the Adomian method and the Successive over relaxation method. Dehghan et al. [5] proposed some new methods for solving fully fuzzy linear systems based on the Cramer's

\footnotetext{
*Corresponding author. nasseri@umz.ac.ir (Hadi Nasseri)
} 
method, Gaussian elimination and LU decomposition method. In this paper, we introduce a linear system of equations with trapezoidal fuzzy numbers as an extension of the common fuzzy linear system of equations. Then we shall describe how can find a fuzzy solution which is including the trapezoidal numbers. In particular, we propose a practical approach for solving these systems. Finally we shall illustrate the process of solving these systems.

\section{Preliminaries}

We represent an arbitrary fuzzy number by an ordered pair of functions $(\underline{u}(r), \bar{u}(r))$, $0 \leq r \leq 1$ which satisfy the following requirements (as given in [6]):

1. $\underline{u}(r)$ is a bounded left continuous nondecreasing function over $[0,1]$.

2. $\bar{u}(r)$ is a bounded left continuous nonincreasing function over $[0,1]$.

3. $\underline{u}(r) \leq \bar{u}(r), 0 \leq r \leq 1$.

For every fuzzy number $x=(\underline{x}(r), \bar{x}(r)), y=(\underline{y}(r), \bar{y}(r))$ and real number $k$,

(a) $x=y$ if and only if $\underline{x}(r)=\underline{y}(r)$ and $\bar{x}(r)=\bar{y}(r)$.

(b) $x+y=(\underline{x}(r)+\underline{y}(r), \bar{x}(r)+\bar{y}(r))$.

(c) $k x= \begin{cases}(k \underline{x}, k \bar{x}), & k \geq 0, \\ (k \bar{x}, k \underline{x}), & k<0 .\end{cases}$

Definition 1. The linear system

$$
\begin{gathered}
a_{11} x_{1}+a_{12} x_{2}+\cdots+a_{1 n} x_{n}=y_{1} \\
a_{21} x_{1}+a_{22} x_{2}+\cdots+a_{2 n} x_{n}=y_{2} \\
\vdots \\
a_{n 1} x_{1}+a_{n 2} x_{2}+\cdots+a_{n n} x_{n}=y_{n}
\end{gathered}
$$

where the coefficient matrix $A=\left(a_{i j}\right), 1 \leq i, j \leq n$ is a $n \times n$ crisp matrix and $y_{i}, 1 \leq i \leq n$ are fuzzy numbers, is called a fuzzy linear system (FLS).

Definition 2. A fuzzy number vector $x=\left(x_{1}, x_{2}, \cdots, x_{n}\right)^{T}$, where

$$
x_{i}=\left(\underline{x}_{i}(r), \bar{x}_{i}(r)\right), \quad 1 \leq i \leq n, 0 \leq r \leq 1,
$$

is called a solution of fuzzy linear system if:

$$
\begin{aligned}
& \frac{\sum_{j=1}^{n} a_{i j} x_{j}}{\overline{\sum_{j=1}^{n} a_{i j} x_{j}}}=\sum_{j=1}^{n} \underline{a_{i j} x_{j}}=\underline{y}_{i}, \\
& \overline{a_{i j} x_{j}}=\bar{y}_{i} .
\end{aligned}
$$


Now, let $s_{i j}, 1 \leq i \leq n, 1 \leq j \leq n$ is defined as follows:

$$
\begin{gathered}
a_{i j} \geq 0 \Rightarrow s_{i j}=a_{i j}, s_{i+n, j+n}=a_{i j} \\
a_{i j}<0 \Rightarrow s_{i, j+n}=-a_{i j}, s_{i+n, j}=-a_{i j}
\end{gathered}
$$

and any $s_{i j}$ that is not determined by $(3)$ is zero. Then (2) is equivalent to the system

$$
S X=Y
$$

where $S=\left(s_{i j}\right), s_{i j} \geq 0,1 \leq i, j \leq 2 n$, and

$$
X=\left[\begin{array}{c}
\underline{x}_{1} \\
\underline{x}_{2} \\
\vdots \\
\underline{x}_{n} \\
-\bar{x}_{1} \\
-\bar{x}_{2} \\
\vdots \\
-\bar{x}_{n}
\end{array}\right]=\left[\begin{array}{c}
\underline{x} \\
-\bar{x}
\end{array}\right], \quad Y=\left[\begin{array}{c}
\underline{y}_{1} \\
\underline{y}_{2} \\
\vdots \\
\underline{y}_{n} \\
-\bar{y}_{1} \\
-\bar{y}_{2} \\
\vdots \\
-\bar{y}_{n}
\end{array}\right]=\left[\begin{array}{c}
\underline{y} \\
-\bar{y}
\end{array}\right] .
$$

In fact, the structure of $S$ implies that

$$
S=\left[\begin{array}{ll}
B & C \\
C & B
\end{array}\right]
$$

where $B$ contains the positive entries of $A, C$ the absolute values of the negative entries of $A$ and $A=B-C$.

In the following we only consider triangular fuzzy numbers, .i.e. $\underline{y}_{i}(r), \bar{y}_{i}(r)$ and consequently $\underline{x}_{i}(r), \bar{x}_{i}(r)$ are all linear functions of $r$. Let $X$ satisfies (4), then the fuzzy solution of (10) is defined in the below.

Theorem 3. The matrix $S$ is nonsingular if and only if the matrices $A=B-C$ and $B+C$ are both nonsingular.

Proof. The same as the proof of Theorem 1 in [6].

the next result is taken from the theory of block matrices and provides the structure of $S^{-1}$.

Theorem 4. if $S^{-1}$ exists it must have the same structure as $S$, i.e.

$$
S^{-1}=\left(\begin{array}{cc}
D & E \\
E & D
\end{array}\right),
$$

Proof. The same as the proof of Theorem 2 in [6]. by assume that $S$ is nonsingular we obtain

$$
X=S^{-1} Y
$$


the following result provides necessary and sufficient conditions for the unique solution vector to be a fuzzy vector, given arbitrary input fuzzy vector $Y$.

Theorem 5. the unique solution $X$ of Eq.(5) is a fuzzy vector for arbitrary $Y$ if and only if $S^{-1}$ is nonnegative, i.e.

$$
\left(S^{-1}\right)_{i j} \geq 0, \quad 1 \leq i, j \leq 2 n
$$

Proof. The same as the proof of Theorem 3 in [6].

Definition 6. Let $X=\left\{\left(\underline{x}_{i}(r), \bar{x}_{i}(r)\right), 1 \leq i \leq n\right\}$ denotes the unique solution of (10). The fuzzy number vector $u=\left\{\left(\underline{u}_{i}(r), \bar{u}_{i}(r)\right), 1 \leq i \leq n\right\}$ defined by

$$
\begin{aligned}
& \underline{u}_{i}(r)=\min \left\{\underline{x}_{i}(r), \bar{x}_{i}(r), x_{i}(1)\right\} \\
& \bar{u}_{i}(r)=\max \left\{\underline{x}_{i}(r), \bar{x}_{i}(r), x_{i}(1)\right\}
\end{aligned}
$$

is called the fuzzy solution of $S X=Y$. Moreover if $\left(\underline{x}_{i}(r), \bar{x}_{i}(r)\right), 1 \leq i \leq n$ are all fuzzy numbers then we have $\underline{x}_{i}(r)=\underline{u}_{i}(r), \bar{x}_{i}(r)=\bar{u}_{i}(r), 1 \leq i \leq n$, and $u$ is called a strong fuzzy solution. Otherwise, $u$ is a week fuzzy solution.

\section{Linear system of equations with trapezoidal fuzzy numbers}

The following definition is concerning to a new achievement to the fuzzy linear systems.

Definition 7. The linear system

$$
\begin{gathered}
a_{11} \tilde{x}_{1}+a_{12} \tilde{x}_{2}+\cdots+a_{1 n} \tilde{x}_{n}=\tilde{y}_{1} \\
a_{21} \tilde{x}_{1}+a_{22} \tilde{x}_{2}+\cdots+a_{2 n} \tilde{x}_{n}=\tilde{y}_{2} \\
\vdots \\
a_{n 1} \tilde{x}_{1}+a_{n 2} \tilde{x}_{2}+\cdots+a_{n n} \tilde{x}_{n}=\tilde{y}_{n}
\end{gathered}
$$

where the coefficient matrix $A=\left(a_{i j}\right), 1 \leq i, j \leq n$ is an $n \times n$ crisp matrix and $\tilde{y}_{i}=\left(y_{i}^{L}, y_{i}^{U}, y_{i}^{\alpha}, y_{i}^{\beta}\right), 1 \leq i \leq n$ are trapezoidal fuzzy numbers, is called a linear system of equations with trapezoidal fuzzy numbers (TFLS).

Definition 8. A trapezoidal fuzzy number vector $\tilde{x}=\left(\tilde{x}_{1}, \tilde{x}_{2}, \cdots, \tilde{x}_{n}\right)^{T}$, where

$$
\tilde{x}_{i}=\left(x_{i}^{L}, x_{i}^{U}, x_{i}^{\alpha}, x_{i}^{\beta}\right), \quad 1 \leq i \leq n,
$$

is called a solution of TFLS if:

$$
\sum_{j=1}^{n} a_{i j} \tilde{x}_{j}=\tilde{y}_{i} .
$$


In the following definition we give an associated triangular fuzzy number for each trapezoidal fuzzy number which will be used in throughout the paper.

Definition 9. For every trapezoidal fuzzy number $\tilde{a}=\left(a^{L}, a^{U}, a^{\alpha}, a^{\beta}\right)$, we define an associated triangular fuzzy number $\hat{a}=\left(a^{m}, \hat{a}^{\alpha}, \hat{a}^{\beta}\right)$ such that $a^{m}=\frac{a^{L}+a^{U}}{2}, \hat{a}^{\alpha}=a^{\alpha}+\left(a^{m}-a^{L}\right)$ and $\hat{a}^{\beta}=a^{\beta}+\left(a^{U}-a^{m}\right)$.

In fact, the support and the core of the associated triangular fuzzy number are $\left(a^{m}-\hat{a}^{\alpha}, a^{m}+\hat{a}^{\beta}\right)$ and the single point (real number) $a^{m}$, respectively. Note that the support of the trapezoidal fuzzy number $\tilde{a}=\left(a^{L}, a^{U}, a^{\alpha}, a^{\beta}\right)$ and its associated triangular fuzzy number is same.

According to the above definition we may define an associated fuzzy linear system of equations for every TFLS which is defined in (6) as follows:

$$
\begin{gathered}
a_{11} \hat{x}_{1}+a_{12} \hat{x}_{2}+\cdots+a_{1 n} \hat{x}_{n}=\hat{y}_{1} \\
a_{21} \hat{x}_{1}+a_{22} \hat{x}_{2}+\cdots+a_{2 n} \hat{x}_{n}=\hat{y}_{2} \\
\vdots \\
a_{n 1} \hat{x}_{1}+a_{n 2} \hat{x}_{2}+\cdots+a_{n n} \hat{x}_{n}=\hat{y}_{n}
\end{gathered}
$$

where $\hat{y}_{i}=\left(y_{i}^{m}, \hat{y}_{i}{ }^{\alpha}, \hat{y}_{i}{ }^{\beta}\right)$ such that $y_{i}^{m}=\frac{y_{i}^{L}+y_{i}^{U}}{2}, \hat{y}_{i}{ }^{\alpha}=y_{i}^{\alpha}+\left(y_{i}^{m}-y_{i}^{L}\right)$ and $\hat{y}_{i}{ }^{\beta}=y_{i}^{\beta}+\left(y_{i}^{U}-y_{i}^{m}\right)$. We name the above fuzzy linear system as "auxiliary fuzzy linear system" (AFLS). Also according to Definition 6, it is clear that there exist a unique auxiliary fuzzy linear system for every TFLS.

Now we are in place that can describe how to solve the linear system of equations with trapezoidal fuzzy numbers.

For achieving to this objective, we first solve the auxiliary fuzzy linear system (8) by one of the various methods which have proposed for solving these systems (see in $[1,2,3,4,5])$. So if we assume that $\hat{x}=\left(\hat{x}_{1}, \ldots, \hat{x}_{j}, \ldots, \hat{x}_{n}\right)^{T}$ be a weak or strong solution for system (8) such that $\hat{x}_{j}=\left(\hat{x}_{j}^{m}, \hat{x}_{j}^{\alpha}, \hat{x}_{j}^{\beta}\right)$, for all $j=1, \ldots, n$, then we must obtain a trapezoidal fuzzy solution. This aim is also complied by the following process.

For the $i$-th right-hand-side $\tilde{y}_{i}=\left(y_{i}^{L}, y_{i}^{U}, y_{i}^{\alpha}, y_{i}^{\beta}\right)$, we first define

$$
R_{i}=\frac{y_{i}^{U}-y_{i}^{L}}{\left(y_{i}^{U}+y_{i}^{\beta}\right)-\left(y_{i}^{L}-y_{i}^{\alpha}\right)},
$$

and then set

$$
\gamma=\max _{1 \leq i \leq n}\left\{R_{i}\right\}
$$

Now for all $j=1, \ldots, n$ set

$$
\gamma_{j}=\gamma * \min \left\{\hat{x}_{j}^{\alpha}, \hat{x}_{j}^{\beta}\right\}
$$


Then the $j$-th component of the approximated trapezoidal fuzzy solution will be defined as follows:

$$
\bar{x}_{j}=\left(x_{j}^{L}, x_{j}^{U}, x_{j}^{\alpha}, x_{j}^{\beta}\right)
$$

where $x_{j}^{L}=\hat{x}_{j}^{m}-\gamma_{j}, x_{j}^{U}=\hat{x}_{j}^{m}+\gamma_{j}, x_{j}^{\alpha}=\hat{x}_{j}^{\alpha}-\gamma_{j}$ and $x_{j}^{\beta}=\hat{x}_{j}^{\beta}-\gamma_{j}$.

Thus, the support and the core of the mentioned trapezoidal fuzzy numbers are respectively $\left(x_{j}^{L}-x_{j}^{\alpha}, x_{j}^{U}+x_{j}^{\beta}\right)$ and $\left[x_{j}^{L}, x_{j}^{U}\right]$.

Now based on the above discussion we can propose the following algorithm for solving TFLSs.

\section{Algorithm 3.1 (TFLS Solver):}

Assumption The $i-t h$ component of the right-hand-side of TFLS is given by $\tilde{y}_{i}=\left(y_{i}^{L}, y_{i}^{U}, y_{i}^{\alpha}, y_{i}^{\beta}\right)$, for all $i=1, \ldots, n$.

Step 1: For every $i=1, \ldots, n$, compute the parameters $y_{i}^{m}, \hat{y}_{i}{ }^{\alpha}, \hat{y}_{i}{ }^{\beta}$ and $R_{i}$ by $y_{i}^{m}=\frac{y_{i}^{L}+y_{i}^{U}}{2}, \hat{y}_{i}{ }^{\alpha}=y_{i}^{\alpha}+\left(y_{i}^{m}-y_{i}^{L}\right), \hat{y}_{i}{ }^{\beta}=y_{i}^{\beta}+\left(y_{i}^{U}-y_{i}^{m}\right)$, and also $R_{i}=\frac{y_{i}^{U}-y_{i}^{L}}{\left(y_{i}^{U}+y_{i}^{\beta}\right)-\left(y_{i}^{L}-y_{i}^{\alpha}\right)}$.

Step 2: Solve the fuzzy linear system (8) by each FLS solver and assume that the $i-t h$ component of the triangular fuzzy solution is $\hat{x}_{i}=\left(\hat{x}_{i}^{m}, \hat{x}_{i}^{\alpha}, \hat{x}_{i}^{\beta}\right)$.

Step 3: Compute the parameter $\gamma_{i}$, for all $i=1, \ldots, n$ by

$$
\gamma_{i}=\gamma * \min \left\{\hat{x}_{i}^{\alpha}, \hat{x}_{i}^{\beta}\right\}
$$

where

$$
\gamma=\max _{1 \leq i \leq n}\left\{R_{i}\right\}
$$

Step 4: Compute the $i-t h$ component of the approximated trapezoidal fuzzy solution by

$$
\bar{x}_{i}=\left(x_{i}^{L}, x_{i}^{U}, x_{i}^{\alpha}, x_{i}^{\beta}\right),
$$

where $x_{i}^{L}=\hat{x}_{i}^{m}-\gamma_{i}, x_{i}^{U}=\hat{x}_{i}^{m}+\gamma_{i}, x_{i}^{\alpha}=\hat{x}_{i}^{\alpha}-\gamma_{i}$ and $x_{i}^{\beta}=\hat{x}_{i}^{\beta}-\gamma_{i}$, and then stop.

\section{Numerical examples}

Here, we give some illustrative examples to show how Algorithm 3.1 solves the TFLSs. 
Example 10. Consider the following TFLS

$$
\begin{gathered}
\tilde{x}_{1}-\tilde{x}_{2}=\tilde{y}_{1}=(-1,3,30,27) \\
\tilde{x}_{1}+5 \tilde{x}_{2}=\tilde{y}_{2}=(1,13,66,87)
\end{gathered}
$$

The associated fuzzy linear system of equations for the above TFLS is as follows:

$$
\begin{gathered}
\hat{x}_{1}-\hat{x}_{2}=\hat{y}_{1}=\left(\underline{y}_{1}(r), \bar{y}_{1}(r)\right)=(-31+32 r, 30-29 r), \\
\hat{x}_{1}+5 \hat{x}_{2}=\hat{y}_{2}=\left(\underline{y}_{2}(r), \bar{y}_{2}(r)\right)=(-65+72 r, 100-93 r) .
\end{gathered}
$$

The strong fuzzy solution of the current fuzzy linear system is as following:

$$
\begin{aligned}
& \hat{x}_{1}=\left(\underline{x}_{1}(r), \bar{x}_{1}(r)\right)=(-15+17 r, 20-18 r), \\
& \hat{x}_{2}=\left(\underline{x}_{2}(r), \bar{x}_{2}(r)\right)=(-10+11 r, 16-15 r) .
\end{aligned}
$$

Thus the approximated trapezoidal fuzzy solution is as follows:

$$
\bar{x}_{1}=(0.82,3.18,15.82,16.82), \quad \bar{x}_{2}=(0.02,1.98,10.02,14.02) .
$$

Note that the trapezoidal fuzzy solution of the mentioned TFLS is as follows:

$$
\tilde{x}_{1}=(1,3,16,17), \quad \tilde{x}_{2}=(0,2,10,14) .
$$

Example 11. Consider the following TFLS

$$
\begin{gathered}
2 \tilde{x}_{1}+\tilde{x}_{2}+3 \tilde{x}_{3}=\tilde{y}_{1}=(7,13,6,6) \\
4 \tilde{x}_{1}+\tilde{x}_{2}-\tilde{x}_{3}=\tilde{y}_{2}=(6,12,6,6) \\
-\tilde{x}_{1}+\tilde{x}_{2}+3 \tilde{x}_{3}=\tilde{y}_{3}=(0,5,5,5)
\end{gathered}
$$

The associated fuzzy linear system of equations for the above TFLS is as follows:

$$
\begin{gathered}
2 \hat{x}_{1}+\hat{x}_{2}+3 \hat{x}_{3}=\hat{y}_{1}=\left(\underline{y}_{1}(r), \bar{y}_{1}(r)\right)=(1+9 r, 19-9 r), \\
4 \hat{x}_{1}+\hat{x}_{2}-\hat{x}_{3}=\hat{y}_{2}=\left(\underline{y}_{2}(r), \bar{y}_{2}(r)\right)=(9 r, 18-9 r), \\
-\hat{x}_{1}+\hat{x}_{2}+3 \hat{x}_{3}=\hat{y}_{3}=\left(\underline{y}_{3}(r), \bar{y}_{3}(r)\right)=\left(-5+\frac{15}{2} r, 10-\frac{15}{2} r\right) .
\end{gathered}
$$

The strong fuzzy solution of the current fuzzy linear system is as following:

$$
\begin{aligned}
& \hat{x}_{1}=\left(\underline{x}_{1}(r), \bar{x}_{1}(r)\right)=\left(1+\frac{3}{2} r, 4-\frac{3}{2} r\right)=\left(\frac{5}{2}, \frac{3}{2}, \frac{3}{2}\right), \\
& \hat{x}_{2}=\left(\underline{x}_{2}(r), \bar{x}_{2}(r)\right)=\left(-1+\frac{3}{2} r, 2-\frac{3}{2} r\right)=\left(\frac{1}{2}, \frac{3}{2}, \frac{3}{2}\right), \\
& \hat{x}_{3}=\left(\underline{x}_{3}(r), \bar{x}_{3}(r)\right)=\left(\frac{3}{2} r, 3-\frac{3}{2} r\right)=\left(\frac{3}{2}, \frac{3}{2}, 3\right) .
\end{aligned}
$$


Thus the approximated trapezoidal fuzzy solution by the 4-th step of Algorithm 3.1 is as follows:

$$
\begin{gathered}
\bar{x}_{1}=\hat{x}_{1}=(2,3,1,1)=\left(\underline{x}_{1}(r), \bar{x}_{1}(r)\right)=(1+r, 4-r), \\
\bar{x}_{2}=\hat{x}_{2}=(0,1,1,1)=\left(\underline{x}_{2}(r), \bar{x}_{2}(r)\right)=(-1+r, 2-r), \\
\bar{x}_{3}=\hat{x}_{3}=(1,2,1,1)=\left(\underline{x}_{3}(r), \bar{x}_{3}(r)\right)=(r, 3-r) .
\end{gathered}
$$

Note that the exact trapezoidal fuzzy solution of the mentioned TFLS is as follows:

$$
\tilde{x}_{1}=(2,3,1,1), \quad \tilde{x}_{2}=(0,1,1,1), \quad \tilde{x}_{3}=(1,2,1,1) .
$$

Example 12. Consider the following TFLS

$$
\begin{aligned}
& \tilde{x}_{1}+\tilde{x}_{2}-\tilde{x}_{3}=\tilde{y}_{1}=(6,11,4,4) \\
& \tilde{x}_{1}-2 \tilde{x}_{2}+\tilde{x}_{3}=\tilde{y}_{2}=(-11,-5,4,7) \\
& 2 \tilde{x}_{1}+\tilde{x}_{2}+3 \tilde{x}_{3}=\tilde{y}_{3}=(-7,4,7,8)
\end{aligned}
$$

The associated fuzzy linear system of equations for the above TFLS is as follows:

$$
\begin{aligned}
\hat{x}_{1}+\hat{x}_{2}-\hat{x}_{3}=\hat{y}_{1}=\left(\underline{y}_{1}(r), \bar{y}_{1}(r)\right)=\left(2+\frac{13}{2} r, 15-\frac{13}{2} r\right)=\left(\frac{17}{2}, \frac{13}{2}, \frac{13}{2}\right), \\
\hat{x}_{1}-2 \hat{x}_{2}+\hat{x}_{3}=\hat{y}_{2}=\left(\underline{y}_{2}(r), \bar{y}_{2}(r)\right)=(-15+7 r, 2-10 r)=(-8,7,10), \\
2 \hat{x}_{1}+\hat{x}_{2}+3 \hat{x}_{3}=\hat{y}_{3}=\left(\underline{y}_{3}(r), \bar{y}_{3}(r)\right)=\left(-14+\frac{25}{2} r, 12-\frac{27}{2} r\right)=\left(-\frac{3}{2}, \frac{25}{2}, \frac{27}{2}\right) .
\end{aligned}
$$

The strong fuzzy solution of the current fuzzy linear system is as following:

$$
\begin{aligned}
& \hat{x}_{1}=\left(\underline{x}_{1}(r), \bar{x}_{1}(r)\right)=(2 r, 5-3 r)=(2,2,3), \\
& \hat{x}_{2}=\left(\underline{x}_{2}(r), \bar{x}_{2}(r)\right)=\left(1+\frac{5}{2} r, 5-\frac{3}{2} r\right)=\left(\frac{7}{2}, \frac{5}{2}, \frac{3}{2}\right), \\
& \hat{x}_{3}=\left(\underline{x}_{3}(r), \bar{x}_{3}(r)\right)=(-5+2 r,-1-2 r)=(-3,2,2) .
\end{aligned}
$$

Thus the approximated trapezoidal fuzzy solution is as follows:

$$
\begin{aligned}
& \bar{x}_{1}=\left(\underline{x}_{1}(r), \bar{x}_{1}(r)\right)=(0.94,3.06,0.94,1.94), \\
& \bar{x}_{2}=\left(\underline{x}_{2}(r), \bar{x}_{2}(r)\right)=(2.62,4.38,1.62,0.62), \\
& \bar{x}_{3}=\left(\underline{x}_{3}(r), \bar{x}_{3}(r)\right)=(-3.71,-2.29,1.29,1.29) .
\end{aligned}
$$

Note that the exact trapezoidal fuzzy solution of the mentioned TFLS is as follows:

$$
\tilde{x}_{1}=(1,3,1,2), \quad \tilde{x}_{2}=(3,4,2,1), \quad \tilde{x}_{3}=(-4,-2,1,1) .
$$




\section{Conclusion}

In this paper, we introduced the trapezoidal fuzzy linear system of equations. For solving these systems we proposed a new algorithm based on solving the associated fuzzy linear system (the auxiliary fuzzy linear system). We also emphasized that there exist a unique associated auxiliary fuzzy linear system of equations for every TFLS. Here according to our suggestion we may obtain a unique trapezoidal fuzzy solution for the given TFLS. We examined some numerical examples to show how solve these systems by Algorithm 3.1.

\section{Achknowledgment}

This work was supported in part by the Research Center of Algebraic Hyperstructures and Fuzzy Mathematics, Babolsar, Iran and also National Elite Foundation, Tehran, Iran. The authors kindly appreciate from their supports.

\section{References}

[1] S. Abbasbandy and M. Alavi, A Method for Solving Fuzzy Linear Systems, Iranian Journal of Fuzzy Systems, 2(2) (2005) 37-44.

[2] T. Allahviranloo, Successive over relaxation iterative method for fuzzy system of linear equation, Applied Mathematics and Computation, 162 (2005) 189-196.

[3] T. Allahviranloo, The adomian decomposition method for fuzzy system of linear equations, Applied Mathematics and Computation, 163 (2005) 553-563.

[4] J.J. Buckley, Y. Qu, Solving systems of linear fuzzy equations, Fuzzy Sets and Systems, 43 (1991) 33-43.

[5] M. Dehghan, B. Hashemi and M. Ghatee, Computational methods for solving fully fuzzy linear systems, Applied Mathematics and Computation, 179 (2006) 328-343.

[6] M. Friedman, M. Ming and A. Kandel, Fuzzy linear systems, Fuzzy Sets and Systems, 96 (1998) 201-209.

[7] S.H. Nasseri, Solving fuzzy linear system of equations by use of the matrix decomposition, International Journal of Applied Mathematics, 21 (2008) 435-445. 